\title{
Regulation of hypoxia adaptation: an overlooked virulence attribute of pathogenic fungi?
}

NORA GRAHL \& ROBERT A. CRAMER JR.

Department of Veterinary Molecular Biology, Montana State University, Bozeman, Montana, USA

Over the past two decades, the incidence of fungal infections has dramatically increased. This is primarily due to increases in the population of immunocompromised individuals attributed to the HIV/AIDS pandemic and immunosuppression therapies associated with organ transplantation, cancer, and other diseases where new immunomodulatory therapies are utilized. Significant advances have been made in understanding how fungi cause disease, but clearly much remains to be learned about the pathophysiology of these often lethal infections. Fungal pathogens face numerous environmental challenges as they colonize and infect mammalian hosts. Regardless of a pathogen's complexity, its ability to adapt to environmental changes is critical for its survival and ability to cause disease. For example, at sites of fungal infections, the significant influx of immune effector cells and the necrosis of tissue by the invading pathogen generate hypoxic microenvironments to which both the pathogen and host cells must adapt in order to survive. However, our current knowledge of how pathogenic fungi adapt to and survive in hypoxic conditions during fungal pathogenesis is limited. Recent studies have begun to observe that the ability to adapt to various levels of hypoxia is an important component of the virulence arsenal of pathogenic fungi. In this review, we focus on known oxygen sensing mechanisms that non-pathogenic and pathogenic fungi utilize to adapt to hypoxic microenvironments and their possible relation to fungal virulence.

Keywords Aspergillus fumigatus, Cryptococcus neoformans, Candida albicans, fungal virulence, hypoxia, sterols

\section{Introduction - Significance of hypoxia during fungal pathogenesis}

Recent advances in medical therapies, organ transplantation, HIV infections, and an increasing geriatric population have generated rising populations of immunocompromised patients. These events have all resulted in a significant increase in life-threatening human fungal infections over the last two decades [1]. The limited treatment options and

Received 6 January 2009; Final revision received 2 April 2009; Accepted 4 April 2009

Correspondence: Robert A. Cramer Jr., PO Box 173610, Bozeman, MT 59717, USA. Tel: +1 406994 7467; Fax: +1 406994 4303; E-mail: rcramer@montana.edu high mortality rates associated with these infections has consequently led to a concerted effort to better understand mechanisms of fungal pathogenesis in mammals. The general rationale behind these studies is that a better understanding of how these organisms cause disease will allow us to develop better technologies for the treatment and prevention of these often lethal infections. One increasing area of fungal pathogenesis research is related to identifying and understanding the basic metabolic pathways utilized by these fungi to survive in the harsh and highly variable mammalian host environment.

The three main fungal pathogens that cause human mycoses, Aspergillus fumigatus, Cryptococcus neoformans, and Candida albicans, are typically opportunistic pathogens. These fungi are saprophytic organisms that 
have evolved a unique combination of attributes to survive in their natural environments. Aspergillus fumigatus is typically found in soil and decaying organic material such as compost heaps. Cryptococcus neoformans is typically found in pigeon droppings, soil and certain trees.

Unlike Aspergillus and Cryptococcus species, Candida albicans is rarely found in the soil or external environment. Instead, it is a normal inhabitant of the human microflora. Thus, C.albicans is already highly adapted to the host environment. C. albicans possess the ability to survive in disparate host environments, as illustrated by its ability to colonize diverse areas of the host (oral, vaginal, gastrointestinal areas). Coincidentally, many of these attributes, which allow fungal survival in their natural ecological niches, appear to also allow these fungi to cause disease in immunocompromised hosts. One overlooked environmental selection pressure found in natural environments of all three of the most common fungal pathogens of humans is low oxygen tension. Whether in the soil, a compost pile, pigeon guano, or the gut of a mammal, these fungi must deal with low levels of oxygen.

The focus of this review is on the increasing evidence that pathogenic fungi must adapt to rapidly changing oxygen levels during fungal infections. It is well established that oxygen levels vary throughout the mammalian body depending on numerous factors including tissue type and presence or absence of an inflammatory response. For example, oxygen levels in most mammalian tissues are found to be considerably below atmospheric levels (21\%) [2-4]. Even in the alveoli of healthy lungs, the most oxygen rich organ and site of infection for many fungal pathogens, the oxygen level is around $14 \%$. By the time oxygen reaches the capillaries and diffuses into surrounding tissues its availability is much lower with levels of $2-4 \%$ reported $[5,6]$. In addition, it is well established that at sites of inflammation available oxygen is significantly reduced compared to surrounding tissues [7-9]. Moreover, in inflamed tissues, the blood supply is often interrupted because the vessels are congested with phagocytes or the pathogen itself $[10,11]$. Thus, it seems highly probable that hypoxic microenvironments are generated during fungal infection.

Indeed, we can look no further than the host response to observe that fungal pathogens are likely exposed to severely low oxygen levels during infection. Immune effector cells, such as neutrophils, function effectively in severely hypoxic microenvironments. These and other cells of the host have evolved distinct mechanisms to deal with hypoxic microenvironments generated during microbial infections. Many of these host response mechanisms are dependent upon the global transcription factor, hypoxia inducible factor (HIF) 1 .
HIF 1 is a heterodimeric transcription factor that consists of one of three $\alpha$-subunits (HIF- $1 \alpha$, HIF- $2 \alpha$, and HIF- $3 \alpha$ ) and one $\beta$-subunit (HIF-1 $\beta$ ), and is the central regulator of hypoxic gene expression in mammals (reviewed in $[12,13$ ). Both the degradation and activity of the HIF- $1 \alpha$ subunit are regulated by oxygen-dependent post-translational hydroxyl modifications. Under hypoxic conditions HIF- $1 \alpha$ is not hydroxylated, leading to an accumulation of the HIF-1 $\alpha$ subunit and expression of hypoxia-responsive genes, including those encoding many glycolytic enzymes, erythropoietin, adrenomedullin, and growth factors $[14,15]$.

In a recent study of Acute Respiratory Distress Syndrome (ARDS) and acute inflammatory lung injury, Thiel et al. [16] provided evidence for the importance of hypoxic microenvironments in regulation of host immune responses. ARDS patients are normally treated with a life-saving oxygen therapy, but this therapy may have a dangerous side effect in patients with uncontrolled pulmonary inflammation. Thiel et al. [16] identified a local tissue hypoxia-driven and adenosin A2A receptor (A2AR)-mediated anti-inflammatory mechanism. Their data suggest that oxygenation may lead to elimination of the A2AR-mediated lung tissue-protecting pathway and thereby further exacerbate lung injury. Taken together, the above observations and studies indicate that mammalian immune system responses to microbial infection and inflammation are critically tied to hypoxic microenvironments.

While the role of hypoxia in the immune response to fungal pathogens is relatively unknown, it follows that since immune cells of the host have evolved mechanisms to function in hypoxia, mammalian fungal pathogens like A. fumigatus, C. neoformans and C. albicans are likely exposed to hypoxic conditions during fungal pathogenesis. Indeed, during A. fumigatus infection, our laboratory has recently observed significant increases in HIF- $1 \alpha$ activity as the fungal infection progresses and inflammation and edema increase in the lung (Grahl and Cramer, unpublished data). In addition, a recent study by Brock et al. [17] demonstrated that hypoxia likely occurs in vivo in the lung during A. fumigatus infection. The authors constructed a luciferase-producing bioluminescent A. fumigatus strain, which was not attenuated in virulence in a murine model of invasive aspergillosis. Interestingly, luminescence from the lungs decreased after reaching a maximum at one day post infection, despite the high number of fungal hyphae present in histology examinations. The authors hypothesize that this phenomenon might be due to the severe tissue damage in and through the pulmonary lesions, which likely decrease the oxygen concentration in the lung tissue. Oxygen is essential for the light-producing reaction, and thus the lack of luminescence is likely attributable to the hypoxia at the site of infection [17]. 
Additional evidence that hypoxia may be a key component of the pathophysiology of invasive fungal infections comes from the observation that there are often significant differences in the in vivo and in vitro test results of antifungal drug efficacies. These differences have recently been postulated to be related to hypoxic conditions found in vivo as demonstrated by in vitro antifungal drug efficacy tests conducted in hypoxia $[6,18]$. Furthermore, recent studies have identified genes responsible for regulating fungal response to hypoxia, and some of these pathways have been observed to be essential for fungal virulence of mammals. Consequently, it seems probable that pathogenic fungi possess mechanisms to adapt to hypoxic microenvironments found in vivo during infection.

The purpose of this review is to summarize recent advances in our understanding of mechanisms that human fungal pathogens use to adapt to hypoxic conditions and to highlight the emerging importance of this area of research in the pathogenic fungi. While studies on hypoxia adaptation in these pathogenic fungi are limited, increasing research attention to this important component of their virulence arsenal has revealed similarities and differences with each other and the model yeast S.cerevisiae and S. pombe. Importantly, we should clarify the distinction between mechanisms of hypoxia adaptation and mechanisms of hypoxic growth. It seems clear that these are two distinct biological processes requiring a distinct set of genes and mechanisms. In our literature review, we did not find detailed studies defining these two likely different processes in the fungi. It is likely that periods of adaptation are different for diverse fungi. A close examination of the methodology used in the cited studies indicated that most, if not all, studies are focused on genes allowing the fungi to adapt to hypoxia (i.e., genes expressed/ required in the early phase of exposure to hypoxia (within 48 hours of a switch to hypoxia), rather than genes required for actual fungal growth in these conditions. Where possible, we have attempted to highlight these distinctions. Finally, we have divided the manuscript into sections detailing fungi with Upc2p orthologs and fungi with SREBP orthologs given the emerging importance of these pathways in oxygen sensing and hypoxia adaptation in fungi.

\section{Fungi with the SREBP analogue Upc2p}

\section{Saccharomyces cerevisiae}

S. cerevisiae cells adapt to anaerobic conditions by inducing expression of a large number of genes, called 'hypoxic genes' [19-25]. The hypoxic genes encode oxygenrelated functions in respiration, heme, and membrane biosynthesis that are required at higher levels when molecular oxygen is limited $[20,26]$. For the regulation of hypoxic genes, the cell senses oxygen availability through cellular heme levels [27,28], and recent studies suggest that oxygen availability can also be sensed through cellular sterol levels [29].

\section{Oxygen sensing by heme}

Molecular oxygen is required as a substrate in two consecutive steps of heme biosynthesis catalyzed by the enzymes coproporphyrinogen oxidase and protoporphyrinogen IX oxidase [30]. In the presence of oxygen (aerobic growth), heme accumulates, binds to the transcriptional activator Hap1p (Heme Activator Protein) and causes the formation of a Hap1p homodimer specific for DNA binding to the cis-element. Hap1p is a protein composed of a zinc-finger DNA binding domain at the $\mathrm{N}$-terminus, a dimerization domain, a heme binding domain within the central region, a heme-responsive motif 7 (HRM7), and a transcriptional activation domain at the C-terminus [31-33]. The heme-Hap1p-complex acts as a transcriptional activator of genes containing its recognition site $\left(5^{\prime} \mathrm{CGGN}_{6} \mathrm{CGG}\right)$ [34-36], such as genes involved in respiration (reviewed in [20]).

In addition, the expression of the ROXI (Repressor Of hypoXic genes) gene is activated by the heme-Hap $1 \mathrm{p}$ complex and Rox $1 \mathrm{p}$ accumulates in the cell under aerobic conditions (reviewed in [26]). The Rox1p repressor binds to its recognition site upstream of the hypoxic genes to repress their transcription [37,38]. Rox1p binds to the DNA with its HMG domain and recruits the general repression complex, Tup1/Ssn6, which binds to the Rox1p repression domain (reviewed in [26]). The repression through Rox $1 p$ varies between hypoxic genes that do not have aerobic counterparts, which are expressed at detectable levels at all oxygen concentrations but their expression is higher when oxygen decreases, like HEM13, OLE1, ERG11, and the autorepressed ROXI itself, and hypoxic genes that have an aerobic homologue, like $H M G 1 / \underline{H G 2}, C O X 5 A / \underline{C O X 5 B}$, $A A C 1, A A C 2 / \underline{A A C 3}$, and TIF51A/ANBI (the first gene is aerobic - last and underlined anaerobic). The anaerobic gene is then completely repressed until very low oxygen concentrations are reached [38,39].

For some hypoxic genes, a second DNA binding protein Mot3p enhances Rox1p repression through helping recruit the Tup1p/Ssn6p complex. Two examples are the ANBI (ANaeroBically Induced) gene, encoding a subunit of eukaryotic initiation factor 5 (eIF-5a), an essential translation factor [40], and the HEM13 gene, encoding the enzyme coproporphyrinogen III oxidase, which catalyzes the rate-limiting step in heme biosynthesis [41]. The hypoxic derepression of HEMI3 allows the cell to continue heme

(c) 2010 ISHAM, Medical Mycology, 48, I-I5 
biosynthesis under limited available oxygen. The strongly repressed ANBI gene has one Mot3p and two Rox1p binding sites in its promoter region, while the promoter of the partially repressed HEM13 contains one Rox $1 \mathrm{p}$ and three Mot3p binding sites. The combination of binding sites determines the strength of repression. Multiple Mot3p binding sites plus a single Rox1p binding site are much weaker than multiple Rox $1 \mathrm{p}$ binding sites plus a single Mot3p binding site [42]. Rox1p and Mot3p both interact with Ssn6p of the general repression complex and Rox $1 p$ stabilizes Mot3p binding to DNA through interactions with Tup1p/Ssn6p [42].

Under hypoxic or anaerobic growth conditions, heme levels are reduced. Hap1p still binds to its cognate site, but in the absence of heme, Hap1p forms a biochemically distinct High-Molecular-weight Complex, HMC, which contains Hap1p and four other proteins including Hsp28p and Ydj1p. This complex represses transcription [43]. Consequently, under hypoxic conditions, ROX1 and MOT3 expression is repressed resulting in the activation of hypoxic gene expression [26].

During adaptation to anaerobic conditions, a complex program of cell wall remodeling occurs in yeast. Under anaerobic conditions, major aerobic cell wall mannoproteins, encoded by $C W P 1$ and $C W P 2$, are replaced by their anaerobic counterparts, encoded by the DAN/TIR genes. The DANITIR genes encode a group of eight cell wall mannoproteins that play a significant role in cell wall permeability $[23,44]$. DAN/TIR genes are regulated by heme, sterol levels, and three DNA binding transcription factors. The heme-dependent repressors Rox $1 p$ and Mot $3 p$ function synergistically to efficiently repress DAN/TIR genes under aerobic conditions [45]. In addition, the sterol depletiondependent activator Upc2p acts through a consensus site termed $A R 1$ to induce the expression of DAN/TIR genes in anaerobic conditions [46]. Sertil et al. [47] observed that the histone deacetylase and global repressor Rpd3p is required for the expression of all the DAN/TIR genes and the hypoxic gene ANBI. Moreover, the authors propose that Rpd3p is recruited to the DAN1 promoter under strict anaerobic conditions. The presence of Rpd3p at the promoter counteracts the function of the repressor Mot3p, which leads to stable binding of the activator Upc2p. Upc $2 p$ then recruits the chromatin remodeling complex Swi/Snf to reorganize chromatin, thereby facilitating the binding of the transcriptional machinery that results in the activation of gene expression [47]. Upc2p, together with the transcription factor $\mathrm{Ecm} 22 \mathrm{p}$, is also responsible for basal and induced expression of genes encoding enzymes of ergosterol biosynthesis in yeast (ERG1, ERG2, $E R G 3, E R G 7, E R G 25, E R G 26$, and ERG27), and it has been implicated in the uptake of sterols under hypoxic conditions [48-52].

\section{Oxygen sensing by sterols}

While heme has been thought to be the primary oxygen sensor in S.cerevisiae, recent studies suggest that sterol levels also play an important role. Upc2p and Ecm22p are functionally related to human sterol regulatory element binding protein (SREBP) with an N-terminal transcription factor domain and a C-terminal transmembrane domain. Although S.cerevisiae lacks an ortholog of SREBP, it seems that a potentially analogous oxygen-sensing mechanism exists in budding yeast regulated through Upc2p and Ecm22p. Marie et al. [53] have observed that Upc2p and $E \mathrm{~cm} 22 \mathrm{p}$ are localized outside of the nucleus in sterol replete conditions, but in conditions of sterol depletion localization shifts toward the nucleus. The authors suggest that the $\mathrm{N}$-terminal transcription factor domain is separated from the $\mathrm{C}$-terminal transmembrane domain by proteolytic cleavage and enters the nucleus to activate gene expression, analogous to SREBP regulation of cholesterol biosynthesis in mammals.

Upc2p and Ecm22p both bind a sequence motif known as the sterol regulatory element (SRE) $[48,49,54]$. Nearly one-third of hypoxically induced genes in S.cerevisiae contain at least one potential Upc2p/Ecm22p binding site, suggesting that these transcription factors are major players in the adaptation to hypoxia [55]. The activation of target genes by Upc2p occurs in response to low sterol levels, which can be caused by blocks in ergosterol biosynthesis or by hypoxia. Davies and Rine [29] observed that both $U p c 2 p$ and $E c m 22 p$ require a functional version of Hap1p for basal expression of $E R G 2$, but when sterols are depleted Upc2p is independent of Hap1p, whereas Ecm 22p still depends upon Hap1p for $E R G$ gene activation. ERG2, ERG3, ERG10, DAN2, and DAN4 are activated by Upc2p solely in response to sterol depletion whereas DAN1 and TIRI respond to both sterols and heme [46].

\section{Other oxygen sensing mechanisms}

An additional hypoxic regulatory pathway involving an antagonistic interaction between the Ord1p repressor and the Yap1p factor (a transcriptional activator involved in oxidative stress response) has been discovered in S. cerevisiae and regulates both TIRI and SRPI. The hypoxic response of TIRI/SRPI (both encode cell wall mannoproteins) depends on the absence of heme but is Roxlp-independent. Under aerobic conditions, Ord1p binds to the SRP1 promoter and expression is repressed. When conditions change to hypoxia, Yap1p also binds to the $S R P 1$ promoter, counteracts the Ord1p effect and SRP1 is expressed [56].

Multiple pathways involved in regulating hypoxic and anoxic gene expression in yeast may exist. Studies of several other hypoxic/anaerobic genes including SUT1, 
encoding a putative $\mathrm{Zn}[\mathrm{II}] 2 \mathrm{Cys} 6$-transcription factor that facilitates the uptake and synthesis of sterols under hypoxic conditions [57], GPD2, encoding an isoenzyme of NADdependent glycerol 3-phosphate dehydrogenase [58], and members of the seripauperine (PAU) family, like TIR1 [59] have demonstrated Rox1p-independent hypoxic/anaerobic induction, but the mechanisms by which this occurs are not yet understood.

Another recently described possible mechanism of hypoxia signaling in yeast involves the mitochondrial respiratory chain, the cytochrome c oxidase and reactive oxygen species $[60,61]$. It has been shown that mitochondria from yeast, rat liver, and plants are capable of nitrite $\left(\mathrm{NO}_{2}^{-}\right)$-dependent nitric oxide (NO) synthesis [60,62-66]. This pathway is induced when cells experience hypoxia, and furthermore, Castello et al. 60] suggest that mitochondrially produced $\mathrm{NO}$ functions in a signaling pathway to the nucleus by reacting with the superoxide produced by hypoxic mitochondria [67] to form peroxynitrite $\left(\mathrm{ONOO}^{-}\right)$ that promotes protein tyrosine nitration of specific proteins that may be involved in a signaling pathway to the nucleus. Future research on this mechanism will likely uncover its specific role in hypoxia adaptation.

It seems clear that adaptation to hypoxia is a complex multi-faceted process regulated via the interaction of several different critical metabolic pathways in the cell. The major regulatory pathways discussed above are summarized in Fig. 1 and Table 1. We now turn our attention to other fungi and discuss similarities and differences with these hypoxia adaptation mechanisms in S. cerevisiae. As many of the pathogenic fungi employ different lifestyles than S. cerevisiae, it is still unclear which of these pathways involved in regulating responses to hypoxia in baker's yeast are conserved in fungi that invade mammalian hosts.

\section{Candida albicans}

Candida albicans is an important human fungal pathogen that causes superficial skin infections as well as deepseated infections, suggesting that its ability to switch between normoxia and hypoxia is a major determinant of its virulence [68]. In C.albicans little is known about mechanisms utilized by this yeast to adapt to hypoxic microenvironments. However, our current knowledge suggests that the transcriptional response to hypoxia differs significantly between C.albicans and S. cerevisiae in important aspects. Although both are generally referred to as facultative anaerobes, genetics studies, conditions required for anaerobic growth, and genome analyses seem to suggest that these hemiascomycota yeast respond differently to changes in oxygen levels.

First, a homologue (Rfg1p) of the S. cerevisiae Rox1p has been identified in C.albicans, but $\mathrm{Rfg} 1 \mathrm{p}$ does not play a role in the regulation of hypoxic genes in this pathogenic yeast as in S. cerevisiae (Table 1). Instead, Rfg1p is a transcriptional regulator that controls filamentous growth, and in that role, is critical for C.albicans virulence [69].

Second, S. cerevisiae genes involved in glycolysis and fermentation are not stimulated by hypoxia $[25,70]$, but hypoxia induces these genes and genes involved in hyphal growth in C.albicans while genes of oxidative metabolism are repressed [68]. During normoxic conditions the global transcription factor Efg $1 p$ regulates the expression of genes

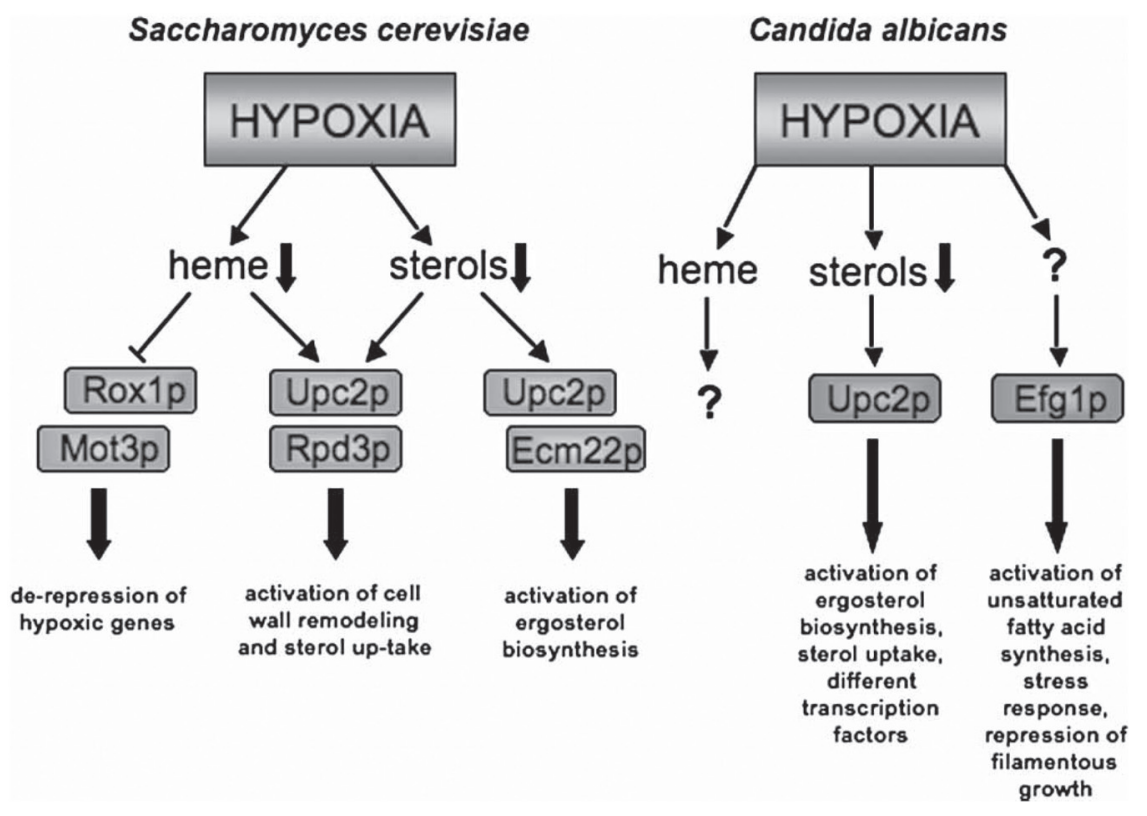

Fig. 1 Schematic of the oxygen sensing pathways in Saccharomyces cerevisiae and Candida albicans. The proteins are defined in the text.

(C) 2010 ISHAM, Medical Mycology, 48, I-I5 

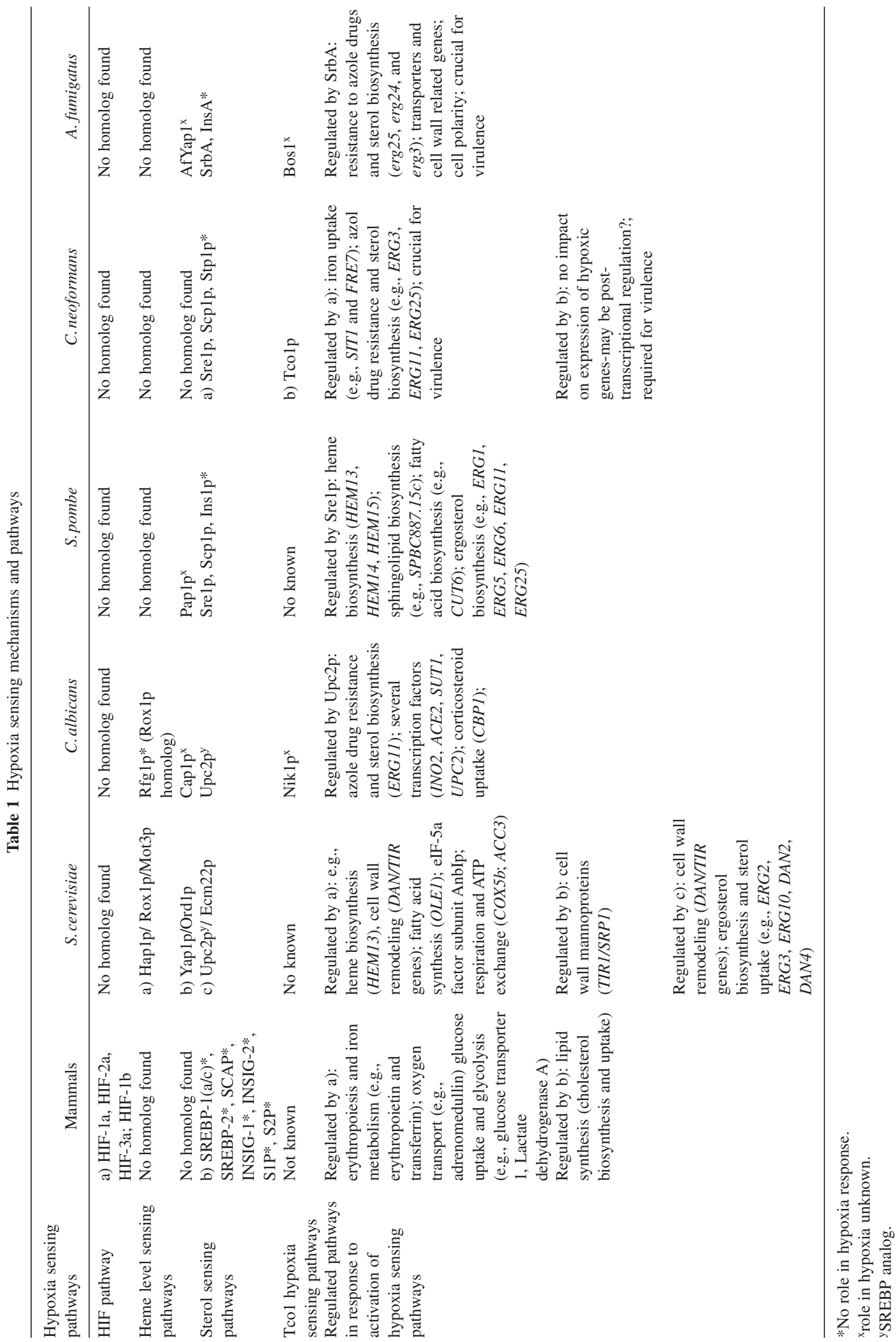
involved in glycolysis and respiration, but it has no role in controlling the expression of respiratory genes and is not required to upregulate glycolytic gene expression in hypoxia [68,71]. Efg1p also promotes filamentation under normoxic conditions. Recently, it was observed that under hypoxic conditions Efg $1 \mathrm{p}$ promotes the synthesis of unsaturated fatty acids, the up-regulation of genes involved in the stress response (HSP12, DDR48, CTA1) and represses filamentous growth in $C$. albicans. Thus, the regulatory role of Efg1p in C.albicans strongly depends on oxygen [68,72]. Transcriptional analyses observed that Efg $1 \mathrm{p}$ is required to allow hypoxic regulation of about half of all genes that are normally regulated by hypoxia in C.albicans. In an efg 1 mutant, hypoxic upregulation (e.g., CTA1) or downregulation (e.g., RIPl) of several genes is abolished, and some genes, like $O L E 1$ encoding a fatty acid desaturase, are ineffectively expressed in hypoxia. Another major function of Efg1p is to prevent hypoxic regulation of numerous genes that are not normally up- or downregulated under hypoxia [71]. Despite the fact that Efg1p is a major regulator of the hypoxic response in C.albicans, a homozygous efg 1 mutant shows no severe change in virulence in comparison to the wild-type [73].

Another transcription factor in C.albicans affected by hypoxia, Ace2p, is required for filamentation in response to hypoxic conditions. Ace $2 p$ also induces fermentative growth and represses respiration, but it is possible that the effect of Ace $2 p$ on metabolism is restricted to normal oxygen conditions. This remains to be tested [74]. Interestingly, an ace 2 null mutant is almost avirulent in an immunocompetent mouse model, while there is only a low degree of attenuation in a neutropenic mouse model [75,76]. This may suggest that different states of the immune system may affect the development of hypoxia in vivo i.e. the lack of neutrophils in the neutropenic model minimizes the inflammatory response and hence hypoxic microenvironments encountered by the invading fungus. This remains to be examined and confirmed.

As in S.cerevisiae [23,44,46], the cell-wall proteome of C.albicans is sensitive to changes in environmental conditions which helps the cell to adjust to harsh environments. For example, iron deprivation and hypoxic conditions affect the expression of cell-wall protein encoding genes, such as iron acquisition and iron-uptake genes, i.e., RBT5 a gene encoding a predicted GPI protein involved in iron acquisition $[68,77,78]$. Numerous oxygendependent reactions in the cell are carried out by ironcontaining enzymes [79]. During hypoxic conditions, there may be competition for iron by iron-containing enzymes, which might lead to an increased expression of cell-wall protein encoding genes involved in iron-acquisition and iron-uptake [80].

\section{Oxygen sensing in Candida albicans}

In S.cerevisiae it has been observed that the cell senses oxygen availability through cellular heme and sterol levels [27-29]. In C.albicans, no apparent homolog of ScHAPl exists, but in recent studies a close ortholog of S. cerevisiae Upc2p and Ecm $22 p$, both involved in sensing sterol depletion, has been identified. Upc2p, a transcription factor of the zinc cluster family, is an important regulator of the sterol biosynthesis and azole drug resistance in C.albicans [81,82] (Fig. 2). Hoot et al. [83] showed that transcriptional regulation of UPC2 expression occurs through Upc2p-dependent as well as a novel Upc2p-independent mechanism. Whether there is also a post-translational control mechanism as described for the mammalian sterol regulator SREBP (discussed in detail below) or as suggested for the $S c \mathrm{Upc} 2 \mathrm{p}$ remains to be determined.

$\mathrm{Upc} 2 \mathrm{p}$ binds in vivo to the promoters of several ergosterol biosynthesis genes and other genes involved or predicted to be involved in lipid metabolism. Znaidi et al. [84] observed that up-regulation of ERG11 during hypoxia is strictly Upc2p dependent. Upc2p also binds the promoters of four genes encoding transcription factors (INO2, $A C E 2$, SUT1, and UPC2 itself). One of them, Sut1p, controls sterol uptake in S. cerevisiae $[57,85]$ suggesting that in C.albicans Upc2p and Sut1p may interact in a sterol regulatory network [84].

Interestingly, Upc $2 \mathrm{p}$ also binds to the promoter of $C B P 1$, which was shown to encode a corticosteroid binding protein in C.albicans [86]. C.albicans appears to take up steroids and possibly metabolic precursors from the host, and Upc $2 p$ seems to play a role in corticosteroid uptake from mammals and in adaptation of $C$.albicans to hypoxic conditions in the host [84]. Thus, an emerging theme with studies in S.cerevisiae and C.albicans is the role of sterol homeostasis in adaptation to hypoxic microenvironments. This theme will also be expanded on in additional fungi discussed below with the discovery of SREBP orthologs. A summary of the known hypoxia regulation mechanisms in C.albicans is presented in Fig. 1 and Table 1.

\section{Fungi with SREBP orthologs}

\section{Schizosaccharomyces pombe}

Recently, a novel mechanism of hypoxia adaptation mediated by a highly conserved family of transcription factors, the SREBPs, was characterized in Schizosaccharomycespombe [87]. S. pombe, also called 'fission yeast', is a non-pathogenic yeast that is used as a model organism in molecular and cell biology. SREBPs are a family of endoplasmic reticulum (ER) membrane bound transcription factors first identified in mammals as regulators of cholesterol and fatty acid synthesis [88-92]. SREBPs contain two 
A

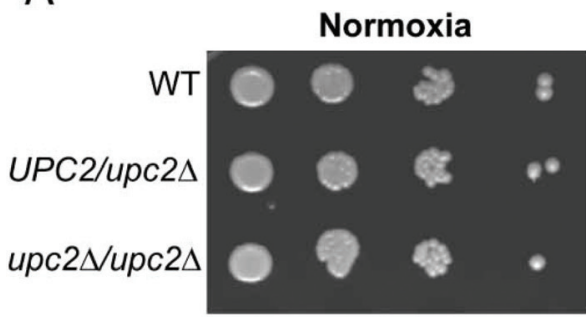

Fig. 2 Mutants in SREBP Pathway and Tco1 are sensitive to hypoxia. Growth in normoxic and hypoxic conditions. (A) Candida albicans: a heterozygous $U P C 2 / u p c 2$ and a homozygous upc $2 \Delta u p c 2 \Delta$ C.albicans strain were serially diluted and spotted on CSM plates and grown at $30^{\circ} \mathrm{C}$. The top panel shows growth in aerobic conditions after $48 \mathrm{~h}$. The bottom panel shows growth in hypoxic conditions after $96 \mathrm{~h}$. Under hypoxic conditions the wild-type (WT) and the heterozygous strain showed comparable growth but the homozygous deletion strain did not demonstrate any detectable growth (Courtesy Chelsea Samaniego and Dr Theodore C. White); (B) Aspergillus fumigatus: $1 \times 10^{6}$ conidia were plated on GMM plates and incubated at $37^{\circ} \mathrm{C}$ under normoxic and hypoxic conditions for $48 \mathrm{~h}$. The wild-type and the reconstituted strain grew comparably under hypoxic conditions while no growth was detectable for the mutant strain (modified from Willger et al. [124]); (C) Cryptococcus neoformans: C.neoformans cultures diluted to $\mathrm{OD}_{600 \mathrm{~nm}}=0.6$ were diluted serially in 10-fold increments prior to being spotted onto YPD plates. The plates were incubated in normoxic or hypoxic conditions in the dark at $37^{\circ} \mathrm{C}$. Under hypoxic conditions all mutants in the SREBP pathway and the tcol $\Delta$ mutants showed reduced growth compared to the wild-type (modified from Chun et al. [100]).
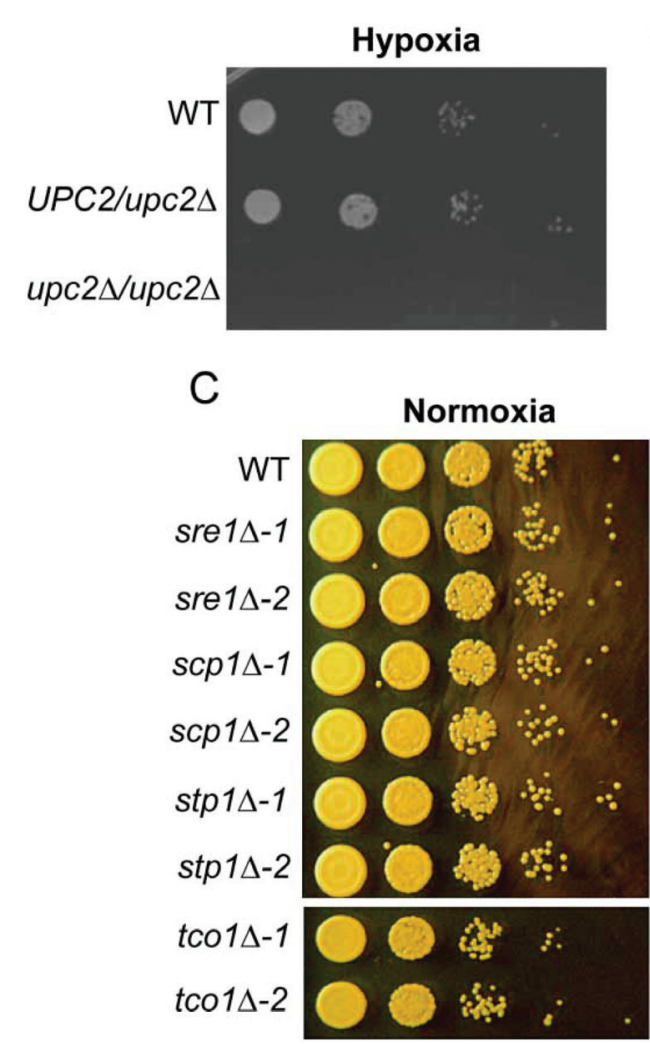

B

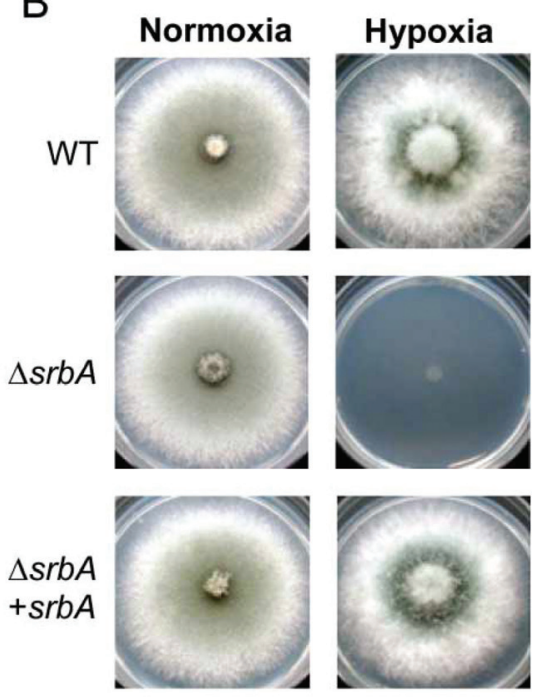

Hypoxia

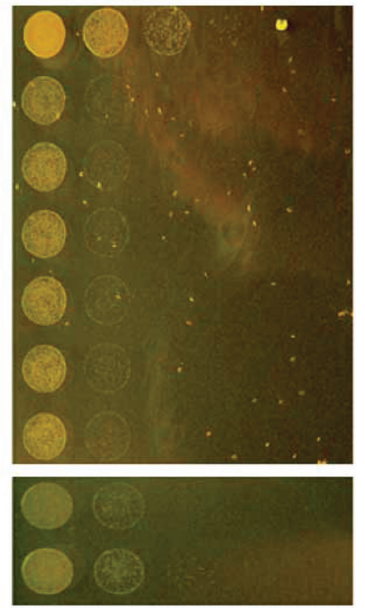

transmembrane segments and are inserted into ER membranes in a hairpin fashion such that the N- and C-terminal ends of the protein are in the cytosol. SREBP is synthesized as an inactive membrane-bound precursor that forms a complex with SCAP (SREBP Cleavage-Acting Protein), a multispan membrane protein that is a component of the sterol sensor [90]. Under conditions with enough available sterols, the SREBP-SCAP complex is retained in the ER membrane through binding of SCAP to the resident ER protein Insig [93]. In sterol-depleted cells, SCAP changes its confirmation, which releases the SREBP-SCAP complex from Insig [94]. SCAP then escorts SREBP from the ER to the Golgi apparatus where SREBP is activated by two sequential proteolytic events catalyzed by site- 1 and site- 2 proteases that release the $\mathrm{N}$-terminal transcription factor domain from the membrane, allowing the transcription factor to enter the nucleus and direct the transcription of target genes [90,95].
In S. pombe apparent orthologs of SREBP (SRE1), SCAP (SCP1) and Insig (INS1) have been identified and characterized. Sre1p is cleaved and activated in response to sterol depletion and hypoxia, and stimulates transcription of genes required for adaptation to hypoxia such as genes involved in heme, sphingolipid, ubiquinone, and ergosterol biosynthesis (Fig. 3 and Table 1) [87,96]. Thus, in fission yeast, Sre1p and Scp1p appear to monitor-oxygen dependent sterol synthesis as an indirect measure of oxygen supply. Interestingly, there does not appear to be an impact of Ins1p on the SREBP pathway in fission yeast. In addition, Hughes and Espenshade [97] recently identified another component of this pathway, Ofd1p. Ofd1p is a prolyl 4-hydroxylase-like 2-oxoglutarate-Fe(II) dioxygenase that accelerates Sre1p degradation in the presence of oxygen. The $\mathrm{N}$-terminal dioxygenase domain is an oxygen sensor that regulates the activity of the C-terminal degradation domain [97]. Altogether, the SREBP pathway 
functions as an oxygen sensor and is required for adaptation to hypoxia in fission yeast. However, the critical function of Sre1 in allowing hypoxic adaptation and subsequent growth is not clearly defined. It seems likely that Sre1 is playing a pleiotropic role in regulating many different genes required for yeast cells to adapt and grow in hypoxia.

Orthologs of the SREBP pathway were recently identified and characterized in the human fungal pathogens C.neoformans and A. fumigatus. Yet, the exact components and the mechanism behind SREBP regulation largely remain to be determined in these pathogenic fungi. Moreover, it appears that the SREBP pathway is similar in function to the Upc2p mediated pathway in S.cerevisiae and C. albicans, but the mechanisms behind the similarities and differences between these two pathways is currently not clear.

\section{Cryptococcus neoformans}

Unlike the Ascomycete yeast S. cerevisiae and C.albicans, the Basidiomycete yeast Cryptococcus neoformans is generally considered an obligate aerobe. Cryptococcus species cause the disease Cryptococcosis in both immunocompromised and apparently healthy hosts and are the most common cause of fungal meningitis [98]. C. neoformans is primarily found in pigeon droppings and soil contaminated with avian guanos throughout the world [99]. One would speculate that these environments are relatively oxygen poor suggesting that C.neoformans likely has evolved mechanisms to adapt to low oxygen microenvironments.
In the laboratory, C.neoformans grows optimally under atmospheric oxygen conditions (21\%), but oxygen concentrations in the human brain are drastically lower than in the atmosphere and vary significantly among anatomical sites [2]. Thus, in order to establish an infection in the brain, it seems likely that $C$.neoformans must adapt to reduced oxygen levels during infection. Therefore, discovering the mechanisms utilized by $C$. neoformans to sense and adapt to low-oxygen conditions is an important area of research aimed towards understanding the pathobiology of this pathogenic yeast. Yet, until recently, the importance of hypoxia adaptation in $C$. neoformans biology and virulence has been largely unstudied.

Recent whole-genome microarray-based transcriptional profiling of $C$. neoformans in a hypoxic microenvironment has started to reveal genes and pathways regulated in response to hypoxia. Among them are genes involved in hexose uptake (sugar transporter and hexose transporter), ethanol production (pyruvate decarboxylase and alcohol dehydrogenases), and sterol metabolism (ergosterol biosynthesis genes) [100]. The possibility of fermentation being important for hypoxic growth during infection is supported by another study, where ethanol was found in cerebral tissue of rats infected with C. neoformans [101]. Yet the importance of fermentation pathways in this obligate aerobe's ability to cause disease and grow in hypoxic microenvironments is unknown. However, as with other yeasts we have discussed, and the filamentous mold A. fumigatus, sterol biosynthesis and homeostasis seems to be a common mechanism regulating adaptation to low oxygen environments in fungi.
Fig. 3 Schematic of the oxygen sensing pathways in Schizosaccharomyces pombe, Cryptococcus neoformans and Aspergillus fumigatus. The proteins are defined in the text.
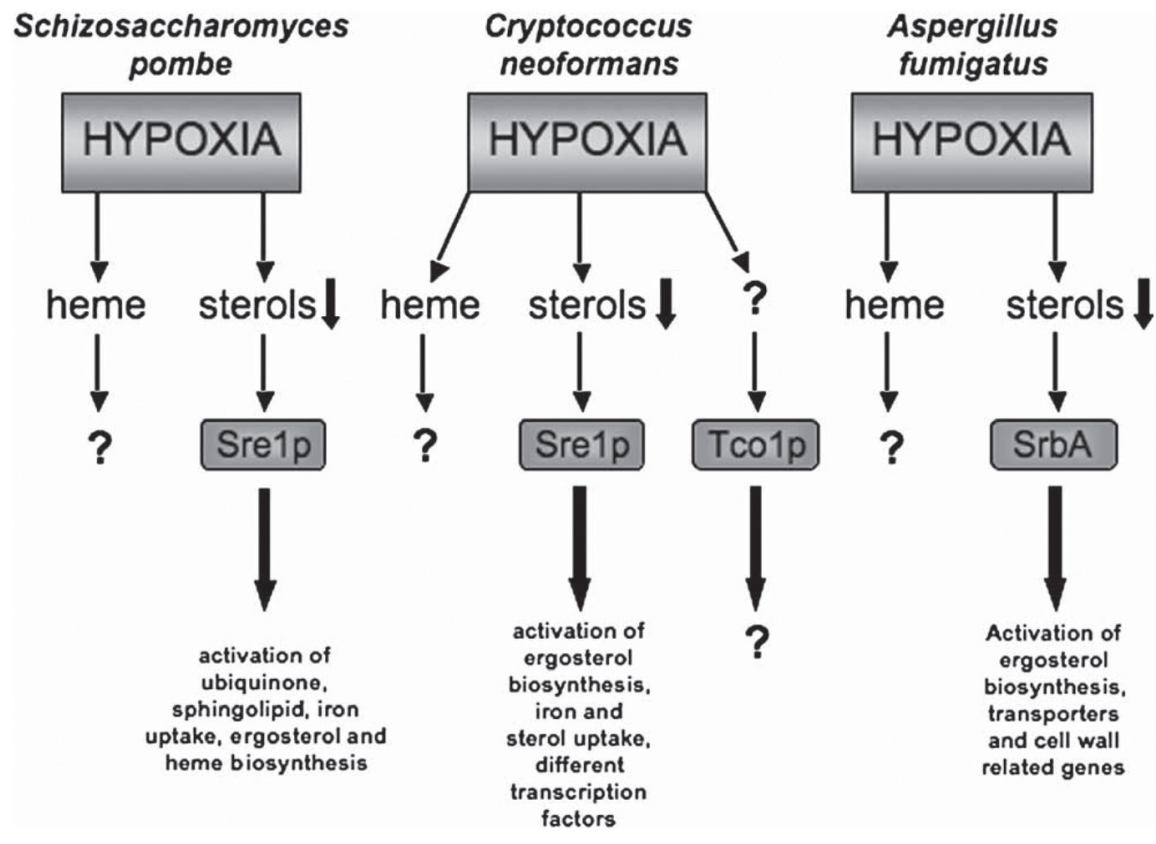


\section{The SREBP pathway in Cryptococcus neoformans}

Orthologs of SREBP (SRE1), SCAP (SCP1) and a Site2-protease $(S T P 1)$ were identified and characterized in C.neoformans $[100,102]$. C. neoformans appears to lack an identifiable homologue of Insig, the ER retention-protein that controls ER-to-Golgi transport of SREBP-SCAP complex in mammalian cells. This finding is consistent with S. pombe data which suggests that Insig is not required for sterol-dependent regulation of Sre1p and Scp1p [87,102]. However, as in fission yeast, the SREBP pathway mediated by Sre1p and Scp1p in C. neoformans is crucial for adaptation to hypoxia and sterol biosynthesis (Fig. 2). In addition, unlike in S. pombe, Sre1p controls low-oxygen expression of genes required for two different pathways of iron uptake (SIT1 and FRE7) [102], which might be crucial for survival under hypoxic conditions. Importantly, sre $1 \Delta$ mutants fail to proliferate in host tissue, fail to cause fatal meningoencephalitis, and display hypersensitivity to the azole class of antifungal drugs (Table 1 and Fig. 3) [100,102]. It is unclear if the virulence defect is due to deficiencies in iron homeostasis, a known virulence attribute of pathogenic fungi, or the inability of C.neoformans to grow in low oxygen microenvironments in the absence of Sre1p. The two phenotypes are likely not mutually exclusive given the importance of iron in ergosterol biosynthesis. However, it is clear that C. neoformans needs Sre1p activation to adapt to the host environment. Mechanisms linking ergosterol biosynthesis, iron homeostasis, and fungal virulence in this pathogenic yeast remain unknown. Identification and characterization of additional components of the SREBP pathway are likely to yield important insights into how this pathogenic yeast causes disease.

In order to identify novel Sre1p pathway components in C.neoformans, Lee et al. [103] observed that responses to cobalt chloride $\left[\mathrm{CoCl}_{2}\right)$ in $C$.neoformans mimic certain aspects of hypoxia by targeting enzymes in the sterol biosynthesis pathway. $\mathrm{CoCl}_{2}$ has been widely used as a hypoxia-mimicking agent in mammalian systems [104-108], but the mechanisms by which it induces hypoxia-mimicking responses are not fully understood. However, Sre1p is required for adaptation to $\mathrm{CoCl}_{2}$ in C.neoformans. Upon $\mathrm{CoCl}_{2}$ treatment, Sre1p is likely activated in response to sterol defects caused by the inhibition of several enzymatic steps in the ergosterol biosynthetic pathway. $\mathrm{CoCl}_{2}$ treatment leads to increased levels of sterol intermediates, including the substrates of Erg25p, 4,4-dimethylfecosterol and 4-methylfecosterol, demonstrating that Sre1p regulates sterol homeostasis in response to $\mathrm{CoCl}_{2}$. $\mathrm{CoCl}_{2}$-induced sterol synthesis inhibition and Sre1p activation has also been observed in S.pombe, suggesting a conserved role for Sre1p in the adaptation to elevated levels of transition metals $[103,109]$.
Consequently, $\mathrm{CoCl}_{2}$ treatment has been used to screen for pathways involved in oxygen sensing in C. neoformans. In this context Ingavale et al. [109] observed that $\mathrm{CoCl}_{2}$ sensitivity and/or oxygen sensing and adaptation processes in C.neoformans have a complex nature. Importantly they identified several mutants with increased sensitivity to $\mathrm{CoCl}_{2}$ and they observed that most of the $\mathrm{CoCl}_{2}$ sensitive mutants are also sensitive to low oxygen concentrations. Mutants included genes involved in the sterol biosynthesis pathway such as SCP1, SRE1, ERG5, mutants in genes involved in mitochondrial function and energy metabolism such as $\mathrm{H}^{+}$transporting ATP synthase, NADH:ubiquinone oxidoreductase or ATP:ADP anitporter, and various transporters and enzymes such as hexose transport related protein, seroheme synthase, amino acid transporter and myo-inositol oxygenase. The role of these genes and pathways in fungal virulence has yet to be explored, but the apparent role of the mitochondia in hypoxia adaptation in C. neoformans echoes the recent findings in $S$. cerevisiae discussed above.

\section{The two-component like (Tco) system in Cryptococcus neoformans}

Additional studies observed that the Sre1p pathway acts in parallel with a two-component signal transduction like pathway controlled by Tcolp in hypoxic adaptation of C. neoformans [100]. Tco1p is a member of a highly conserved family of fungal-specific histidine kinases. Tcolp negatively regulates the expression of melanin formation and, redundantly with Tco2p, positively regulates the HOG MAPK pathway [which is dispensable for virulence) [110]. Interestingly, it has been shown that Tcolp is required for growth under hypoxic conditions and for virulence of C.neoformans (Fig. 2) [100,110]. However, it is unclear how this pathway is involved in hypoxic adaptation and fungal virulence [Fig. 3). In contrast to mutants in SRE1, the tcol mutant shows no detectable defects in the regulation of any of the known hypoxic genes ([100], unpublished data). As a result Chun et al. [100] hypothesize that the Tcolp pathway might act post-transcriptionally. However, this result may also indicate that novel pathways or altered function of existing known pathways regulated by Tco1p are involved in hypoxia adaptation. Clearly, however, these data suggest that oxygen sensing in C.neoformans is highly complex, and likely important for virulence of this organism.

\section{Aspergillus fumigatus}

A. fumigatus is a saprophytic, obligate aerobic filamentous fungus commonly found in soil and compost piles. Its primary ecological function is to recycle carbon and nitrogen 
through the environment [111-113]. As with most fungi, it seems self-evident that these microenvironments would place significant oxygen related stress on the mold. While A. fumigatus is responsible for a number of clinically relevant diseases, invasive pulmonary aspergillosis (IPA) is the most lethal with mortality rates ranging from $60-90 \%$ [114-116]. Interestingly, while IPA can be caused by several Aspergillus species, the majority of IPA cases are caused by A. fumigatus. This may suggest that A. fumigatus contains unique attributes that allow it to cause disease [117].

Currently, we have a limited understanding of the in vivo growth mechanisms of A. fumigatus during IPA [118]. Given that the lung is the primary site of infection for this mold, it may be counter-intuitive to think that low oxygen levels would be a critical component of the pathophysiology of IPA. However, during infection, A. fumigatus causes significant damage to host tissue through invasive growth by hyphae and subsequent recruitment of immune effector cells (depending on the immune system status of the host). Thus, infection generates significant inflammation and necrosis in lung tissue that can be visualized by histopathology. These pathologic lesions also likely represent areas of poor oxygen availability to the pathogen and host. Thus, it is likely that to cause disease A. fumigatus must adapt to hypoxic conditions.

In general, mechanisms of hypoxia adaptation in molds have gone largely unstudied. Tarrand et al. [119,120] hypothesized that the low rate of Aspergillus recovery from clinical material is due to adaptation by the fungus to the physiologic temperature and hypoxic milieu found in vivo. However, recent studies with A. fumigatus suggest that it cannot grow in anaerobic environments [121]. Interestingly, studies with the relatively non-pathogenic model mold A. nidulans observed that while the mold could not proliferate without oxygen, ethanol fermentation was required for its long-term survival in anaerobic conditions [122,123]. Indeed, analyses of Aspergillus genome sequences have revealed numerous potential fermentation pathways in these molds. Yet, as obligate aerobes, it remains unclear what function these pathways may serve in hypoxia adaptation and fungal virulence. Recent studies in our laboratory have identified ethanol fermentation during A. fumigatus infection in a murine model of IPA indicating that fermentation may be a component of the virulence arsenal of this mold (Grahl et al. unpublished data). However, our knowledge about the mechanisms by which A. fumigatus, an obligate aerobe, adapts to hypoxic environments remains extremely limited.

\section{The SREBP pathway in Aspergillus fumigatus}

Recently, our laboratory identified and characterized an SREBP (Sre1p) ortholog, SrbA, in A. fumigatus [124]. As in
C.neoformans, SrbA is crucial for adaptation to hypoxia, mediates resistance to the azole class of antifungal drugs and is involved in sterol biosynthesis in A. fumigatus (Fig. 2 and Fig. 3). In addition, unlike C.neoformans, but similar to S. pombe, transcriptional profiling of the SrbA null mutant suggested that SrbA does not appear to be involved in iron uptake or homeostasis in A. fumigatus. However, these studies may have been limited by the type of media utilized, and direct studies regarding the role of SrbA in iron homeostasis in this pathogenic mold are ongoing.

Importantly, srbA null mutants are almost avirulent in two distinct murine models of IPA. While our results strongly suggest that the virulence defect is due to the inability of this mutant to grow in hypoxia due to the loss of hypoxia adaptation mechanisms regulated by SrbA, as with C.neoformans, other potential hypotheses may explain the attenuation of virulence [124]. For example, SrbA plays an important role in maintenance of cell polarity in A. fumigatus [124]. We hypothesize that the accumulation of sterol intermediates leads to dysfunction in the formation or localization of sterol microdomains known to be required for maintaining cell polarity. Thus, the alteration of cell polarity may inhibit the ability of the SrbA mutant to cause disease. Yet, the mutant displays a normal growth rate in vitro suggesting that the altered cell polarity does not alter growth in these conditions. Altogether, these data promote the hypothesis that hypoxia plays a key role in the pathophysiology of IPA. At the least, it is apparent that SREBPs are critical components of fungal virulence in both pathogenic yeast and molds.

So far the molecular mechanism behind SrbA regulation and activation in molds is unclear. In the yeast S.pombe and C.neoformans, it seems evident that Sre $1 \mathrm{p}$ is regulated post-translationally in response to sterol biosynthesis perturbation that occurs in low oxygen environments. Indeed, Hughes et al. [125] have identified 4-methyl sterols as the primary activating agent of Sre $1 \mathrm{p}$ in S. pombe and C. neoformans. Thus, our finding that the SrbA null mutant in A. fumigatus accumulates 4-methyl sterols may also suggest that these sterols are the trigger for SrbA activation in A.fumigatus [124].

While many of the phenotypes observed in the A.fumigatus srbA mutant may suggest that $\mathrm{SrbA}$ is regulated in a similar manner as Sre1p in yeast, our results may also suggest an alternative model in molds. For example, despite intense bioinformatic analyses, we have been unable to identify clear homologs of SCAP or the proteases required for Sre $1 p$ activation. We have, however, identified a potential Insig1 homolog (insA) and we are currently characterizing a possible role for InsA in SREBP signaling in filamentous fungi. Yet, given the conservation of SCAP across many organisms, it is surprising that an ortholog 
does not appear to be present in the Aspergilli. It may be that another protein with a divergent sequence performs a similar function as SCAP, or it may suggest that a novel mechanism of SREBP regulation and activation exists in molds. Studies to examine these potential mechanisms are ongoing in our laboratory.

\section{Conclusion}

In this review we have attempted to survey the known mechanisms utilized by fungi to regulate adaptation to hypoxic microenvironments. It is clear that we are just beginning to understand the mechanisms human fungal pathogens use to survive in vivo during infection. With the possible exception of SREBPs, the molecular mechanisms utilized by pathogenic fungi to adapt to hypoxic microenvironments found at sites of infection remain to be elucidated. A master regulator of hypoxia adaptation, such as HIF1 found in mammals, has not been identified in fungi. It remains to be seen whether one exists, or, if as suggested by current data, fungi rely on multiple mechanisms to sense oxygen levels and adapt to low oxygen environments.

In any case, we feel that this area of pathogenic fungal physiology has been ignored for too long. Certainly, some mechanisms of hypoxia adaptation, perhaps such as heme biosynthesis, are likely to be conserved between S. cerevisiae and the human pathogenic fungi. However, the different life-styles and selection pressures on nonpathogenic and pathogenic fungi likely have resulted in unique mechanisms of hypoxia adaptation. Thus, solely relying on S.cerevisiae as a model for how pathogenic regulate adaptation to hypoxic microenvironments is likely not appropriate. Regardless, it seems clear that mechanisms of hypoxia adaptation have important implications for fungal virulence and how we manage and treat invasive fungal infections. Therefore, future studies on discovering the conserved and unique pathways utilized by the major fungal pathogens of humans to adapt to hypoxia are likely to yield important insights into sterol metabolism, fungal growth, mechanisms of drug resistance, and fungal virulence.

\section{Acknowledgements}

RAC is currently supported by funding from the National Institutes of Health, COBRE grant RR020185, and the Montana State University Agricultural Experiment Station.

Declaration of interest: The authors report no conflicts of interest. The authors alone are responsible for the content and writing of the paper.

\section{References}

1 McNeil MM, Nash SL, Hajjeh RA, et al. Trends in mortality due to invasive mycotic diseases in the United States, 1980-1997. Clin Infect Dis 2001; 33: 641-647.

2 Erecinska M, Silver IA. Tissue oxygen tension and brain sensitivity to hypoxia. Respir Physiol 2001; 128: 263-276.

3 Carlsson PO, Palm F, Andersson A, Liss P. Markedly decreased oxygen tension in transplanted rat pancreatic islets irrespective of the implantation site. Diabetes 2001; 50: 489-495.

4 Studer L, Csete M, Lee SH, et al. Enhanced proliferation, survival, and dopaminergic differentiation of CNS precursors in lowered oxygen. J Neurosci 2000; 20: 7377-7383.

5 West JB. Respiratory Physiology - The Essentials, 3 edn. Baltimore, MD, USA: Williams \& Wilkins, 1985.

6 Warn PA, Sharp A, Guinea J, Denning DW. Effect of hypoxic conditions on in vitro susceptibility testing of amphotericin B, itraconazole and micafungin against Aspergillus and Candida. J Antimicrob Chemother 2004; 53: 743-749.

7 Matherne GP, Headrick JP, Coleman SD, Berne RM. Interstitial transudate purines in normoxic and hypoxic immature and mature rabbit hearts. Pediatr Res 1990; 28: 348-353.

8 Van Belle H, Goossens F, Wynants J. Formation and release of purine catabolites during hypoperfusion, anoxia, and ischemia. Am J Physiol 1987; 252: (5 Pt 2): H886-H893.

9 Dewhirst MW. Concepts of oxygen transport at the microcirculatory level. Semin Radiat Oncol 1998; 8: 143-150.

10 Arnold F, West D, Kumar S. Wound healing: the effect of macrophage and tumour derived angiogenesis factors on skin graft vascularization. Br J Exp Pathol 1987; 68: 569-574.

11 Simmen HP, Battaglia H, Giovanoli P, Blaser J. Analysis of pH, pO2 and $\mathrm{pCO} 2$ in drainage fluid allows for rapid detection of infectious complications during the follow-up period after abdominal surgery. Infection 1994; 22: 386-389.

12 Schofield CJ, Ratcliffe PJ. Signalling hypoxia by HIF hydroxylases. Biochem Biophys Res Commun 2005; 338: 617-626.

13 Gordan JD, Simon MC. Hypoxia-inducible factors: central regulators of the tumor phenotype. Curr Opin Genet Dev 2007; 17: 71-77.

14 Wenger RH. Cellular adaptation to hypoxia: O2-sensing protein hydroxylases, hypoxia-inducible transcription factors, and O2-regulated gene expression. Faseb J 2002; 16: 1151-1162.

15 Maxwell PH, Dachs GU, Gleadle JM, et al. Hypoxia-inducible factor-1 modulates gene expression in solid tumors and influences both angiogenesis and tumor growth. Proc Natl Acad Sci USA 1997; 94: 8104-8109.

16 Thiel M, Chouker A, Ohta A, et al. Oxygenation inhibits the physiological tissue-protecting mechanism and thereby exacerbates acute inflammatory lung injury. PLoS Biol 2005; 3: e174.

17 Brock M, Jouvion G, Droin-Bergere S, et al. Bioluminescent Aspergillus fumigatus, a new tool for drug efficiency testing and in vivo monitoring of invasive aspergillosis. Appl Environ Microbiol 2008; 74: 7023-7035.

18 Perkhofer S, Jost D, Dierich MP, Lass-Florl C. Susceptibility testing of anidulafungin and voriconazole alone and in combination against conidia and hyphae of Aspergillus spp. under hypoxic conditions. Antimicrob Agents Chemother 2008; 52: 1873-1875.

19 Lowry CV, Zitomer RS. Oxygen regulation of anaerobic and aerobic genes mediated by a common factor in yeast. Proc Natl Acad Sci USA 1984; 81: 6129-6133.

20 Zitomer RS, Lowry CV. Regulation of gene expression by oxygen in Saccharomyces cerevisiae. Microbiol Rev 1992; 56: 1-11.

21 Choi JY, Stukey J, Hwang SY, Martin CE. Regulatory elements that control transcription activation and unsaturated fatty acid-mediated 
repression of the Saccharomyces cerevisiae OLE1 gene. J Biol Chem 1996; 271: 3581-3589.

22 Evangelista CC, Jr, Rodriguez Torres AM, Limbach MP, Zitomer RS. Rox 3 and Rts 1 function in the global stress response pathway in baker's yeast. Genetics 1996; 142: 1083-1093.

23 Sertil O, Cohen BD, Davies KJ, Lowry CV. The DAN1 gene of S. cerevisiae is regulated in parallel with the hypoxic genes, but by a different mechanism. Gene 1997; 192: 199-205.

24 Kwast KE, Burke PV, Staahl BT, Poyton RO. Oxygen sensing in yeast: evidence for the involvement of the respiratory chain in regulating the transcription of a subset of hypoxic genes. Proc Natl Acad Sci USA 1999; 96: 5446-5451.

25 ter Linde JJ, Liang H, Davis RW, et al. Genome-wide transcriptional analysis of aerobic and anaerobic chemostat cultures of Saccharomyces cerevisiae. J Bacteriol 1999; 181: 7409-7413.

26 Zitomer RS, Carrico P, Deckert J. Regulation of hypoxic gene expression in yeast. Kidney Int 1997; 51: 507-513.

27 Zhang L, Hach A. Molecular mechanism of heme signaling in yeast: the transcriptional activator Hap1 serves as the key mediator. Cell Mol Life Sci 1999; 56: 415-426.

28 Hon T, Dodd A, Dirmeier R, et al. A mechanism of oxygen sensing in yeast. Multiple oxygen-responsive steps in the heme biosynthetic pathway affect Hap1 activity. J Biol Chem 2003; 278: 50771-50780.

29 Davies BS, Rine J. A role for sterol levels in oxygen sensing in Saccharomyces cerevisiae. Genetics 2006; 174: 191-201.

30 Labbe-Bois R, Labbe P. Tetrapyrrole and heme biosynthesis in the yeast Saccharomyces cerevisiae. In: Dailey HA (ed.). Biosynthesis of Heme and Chlorophylls. New York: McGraw-Hill Publishing Co, 1990:235-285.

31 Creusot F, Verdiere J, Gaisne M, Slonimski PP. CYP1 [HAP1) regulator of oxygen-dependent gene expression in yeast. I. Overall organization of the protein sequence displays several novel structural domains. J Mol Biol 1988; 204: 263-276.

32 Pfeifer K, Kim KS, Kogan S, Guarente L. Functional dissection and sequence of yeast HAP1 activator. Cell 1989; 56: 291-301.

33 Zhang L, Guarente L. Heme binds to a short sequence that serves a regulatory function in diverse proteins. Embo J 1995; 14: 313-320.

34 Pfeifer K, Arcangioli B, Guarente L. Yeast HAP1 activator competes with the factor $R C 2$ for binding to the upstream activation site UAS1 of the CYC1 gene. Cell 1987; 49: 9-18.

35 Cerdan ME, Zitomer RS. Oxygen-dependent upstream activation sites of Saccharomyces cerevisiae cytochrome c genes are related forms of the same sequence. Mol Cell Biol 1988; 8: 2275-2279.

36 Zhang L, Guarente L. The yeast activator HAP1 - a GALA family member - binds DNA in a directly repeated orientation. Genes Dev 1994; 8: 2110-2119.

37 Lowry CV, Zitomer RS. ROX1 encodes a heme-induced repression factor regulating ANB1 and CYC7 of Saccharomyces cerevisiae. Mol Cell Biol 1988; 8: 4651-4658.

38 Balasubramanian B, Lowry CV, Zitomer RS. The Rox1 repressor of the Saccharomyces cerevisiae hypoxic genes is a specific DNAbinding protein with a high-mobility-group motif. Mol Cell Biol 1993; 13: 6071-6078.

39 Deckert J, Perini R, Balasubramanian B, Zitomer RS. Multiple elements and auto-repression regulate Rox1, a repressor of hypoxic genes in Saccharomyces cerevisiae. Genetics 1995; 139: 1149-1158.

40 Kang HA, Schwelberger HG, Hershey JW. The two genes encoding protein synthesis initiation factor eIF-5A in Saccharomyces cerevisiae are members of a duplicated gene cluster. Mol Gen Genet. 1992; 233: 487-490.

41 Zagorec M, Labbe-Bois R. Negative control of yeast coproporphyrinogen oxidase synthesis by heme and oxygen. J Biol Chem 1986; 261: 2506-2509.
42 Klinkenberg LG, Mennella TA, Luetkenhaus K, Zitomer RS. Combinatorial repression of the hypoxic genes of Saccharomyces cerevisiae by DNA binding proteins Rox 1 and Mot3. Eukaryot Cell 2005; 4: 649-660.

43 Zhang L, Hach A, Wang C. Molecular mechanism governing heme signaling in yeast: a higher-order complex mediates heme regulation of the transcriptional activator HAP1. Mol Cell Biol 1998; 18: $3819-3828$.

44 Abramova N, Sertil O, Mehta S, Lowry CV. Reciprocal regulation of anaerobic and aerobic cell wall mannoprotein gene expression in Saccharomyces cerevisiae. J Bacteriol 2001; 183: 2881-2887.

45 Sertil O, Kapoor R, Cohen BD, Abramova N, Lowry CV. Synergistic repression of anaerobic genes by Mot3 and Rox 1 in Saccharomyces cerevisiae. Nucleic Acids Res 2003; 31: 5831-5837.

46 Abramova NE, Cohen BD, Sertil O, et al. Regulatory mechanisms controlling expression of the DAN/TIR mannoprotein genes during anaerobic remodeling of the cell wall in Saccharomyces cerevisiae. Genetics 2001; 157: 1169-1177.

47 Sertil O, Vemula A, Salmon SL, Morse RH, Lowry CV. Direct role for the Rpd3 complex in transcriptional induction of the anaerobic DAN/TIR genes in yeast. Mol Cell Biol 2007; 27: 2037-2047.

48 Vik A, Rine J. Upc2p and Ecm22p, dual regulators of sterol biosynthesis in Saccharomyces cerevisiae. Mol Cell Biol 2001; 21: 6395-6405.

49 Germann M, Gallo C, Donahue T, et al. Characterizing sterol defect suppressors uncovers a novel transcriptional signaling pathway regulating zymosterol biosynthesis. J Biol Chem 2005; 280: 35904-35913.

50 Lewis TL, Keesler GA, Fenner GP, Parks LW. Pleiotropic mutations in Saccharomyces cerevisiae affecting sterol uptake and metabolism. Yeast 1988; 4: 93-106.

51 Crowley JH, Leak FW, Jr, Shianna KV, Tove S, Parks LW. A mutation in a purported regulatory gene affects control of sterol uptake in Saccharomyces cerevisiae. J Bacteriol 1998; 180: 4177-4183.

52 Wilcox LJ, Balderes DA, Wharton B, et al. Transcriptional profiling identifies two members of the ATP-binding cassette transporter superfamily required for sterol uptake in yeast. J Biol Chem 2002; 277: 32466-32472.

53 Marie C, Leyde S, White TC. Cytoplasmic localization of sterol transcription factors Upc2p and Ecm22p in S. cerevisiae. Fungal Genet Biol 2008; 45: 1430-1438.

54 Davies BS, Wang HS, Rine J. Dual activators of the sterol biosynthetic pathway of Saccharomyces cerevisiae: similar activation/ regulatory domains but different response mechanisms. Mol Cell Biol 2005; 25: 7375-7385.

55 Kwast KE, Lai LC, Menda N, et al. Genomic analyses of anaerobically induced genes in Saccharomyces cerevisiae: functional roles of Rox 1 and other factors in mediating the anoxic response. $J$ Bacteriol 2002; 184: 250-265.

56 Bourdineaud JP, De Sampaio G, Lauquin GJ. A Rox1-independent hypoxic pathway in yeast. Antagonistic action of the repressor Ord1 and activator Yap1 for hypoxic expression of the SRP1/TIR1 gene. Mol Microbiol 2000; 38: 879-890.

57 Ness F, Bourot S, Regnacq M, et al. SUT1 is a putative Zn[II]2Cys6transcription factor whose upregulation enhances both sterol uptake and synthesis in aerobically growing Saccharomyces cerevisiae cells. Eur J Biochem. 2001; 268: 1585-1595.

58 Ansell R, Granath K, Hohmann S, Thevelein JM, Adler L. The two isoenzymes for yeast NAD+-dependent glycerol 3-phosphate dehydrogenase encoded by GPD1 and GPD2 have distinct roles in osmoadaptation and redox regulation. Embo J 1997; 16: 2179-2187. 
59 Rachidi N, Martinez MJ, Barre P, Blondin B. Saccharomyces cerevisiae PAU genes are induced by anaerobiosis. Mol Microbiol 2000; 35: 1421-1430.

60 Castello PR, David PS, McClure T, Crook Z, Poyton RO. Mitochondrial cytochrome oxidase produces nitric oxide under hypoxic conditions: implications for oxygen sensing and hypoxic signaling in eukaryotes. Cell Metab 2006; 3: 277-287.

61 Castello PR, Woo DK, Ball K, et al. Oxygen-regulated isoforms of cytochrome c oxidase have differential effects on its nitric oxide production and on hypoxic signaling. Proc Natl Acad Sci USA 2008; 105: 8203-8208.

62 Gupta KJ, Stoimenova M, Kaiser WM. In higher plants, only root mitochondria, but not leaf mitochondria reduce nitrite to $\mathrm{NO}$, in vitro and in situ. J Exp Bot 2005; 56: 2601-2609.

63 Nohl H, Staniek K, Sobhian B, et al. Mitochondria recycle nitrite back to the bioregulator nitric monoxide. Acta Biochim Pol 2000; 47: 913-921.

64 Nohl H, Staniek K, Kozlov AV. The existence and significance of a mitochondrial nitrite reductase. Redox Rep 2005; 10: 281-286.

65 Planchet E, Jagadis Gupta K, Sonoda M, Kaiser WM. Nitric oxide emission from tobacco leaves and cell suspensions: rate limiting factors and evidence for the involvement of mitochondrial electron transport. Plant J 2005; 41: 732-743.

66 Tischner R, Planchet E, Kaiser WM. Mitochondrial electron transport as a source for nitric oxide in the unicellular green alga Chlorella sorokiniana. FEBS Lett 2004; 576: 151-155.

67 Dirmeier R, O'Brien KM, Engle M, et al. Exposure of yeast cells to anoxia induces transient oxidative stress. Implications for the induction of hypoxic genes. J Biol Chem 2002; 277: 34773-34784.

68 Setiadi ER, Doedt T, Cottier F, Noffz C, Ernst JF. Transcriptional response of Candida albicans to hypoxia: linkage of oxygen sensing and Efg1p-regulatory networks. J Mol Biol 2006; 361: 399-411.

69 Kadosh D, Johnson AD. Rfg1, a protein related to the Saccharomyces cerevisiae hypoxic regulator Rox1, controls filamentous growth and virulence in Candida albicans. Mol Cell Biol 2001; 21: 2496-2505.

70 Becerra M, Lombardia-Ferreira LJ, Hauser NC, et al. The yeast transcriptome in aerobic and hypoxic conditions: effects of hapl, roxl, rox3 and srb10 deletions. Mol Microbiol 2002; 43: 545-555.

71 Ernst JF, Tielker D. Responses to hypoxia in fungal pathogens. Cell Microbiol. 2008; 11: 183-190.

72 Doedt T, Krishnamurthy S, Bockmuhl DP, et al. APSES proteins regulate morphogenesis and metabolism in Candida albicans. Mol Biol Cell 2004; 15: 3167-3180.

73 Lo HJ, Kohler JR, DiDomenico B, et al. Nonfilamentous C. albicans mutants are avirulent. Cell 1997; 90: 939-949.

74 Mulhern SM, Logue ME, Butler G. Candida albicans transcription factor Ace2 regulates metabolism and is required for filamentation in hypoxic conditions. Eukaryot Cell 2006; 5: 2001-2013.

75 Kelly MT, MacCallum DM, Clancy SD, et al. The Candida albicansCaACE2 gene affects morphogenesis, adherence and virulence. Mol Microbiol 2004; 53: 969-983.

76 MacCallum DM, Findon H, Kenny CC, et al. Different consequences of ACE2 and SWI5 gene disruptions for virulence of pathogenic and nonpathogenic yeasts. Infect Immun 2006; 74: 5244-5248.

77 Lan CY, Rodarte G, Murillo LA, et al. Regulatory networks affected by iron availability in Candida albicans. Mol Microbiol 2004; 53: 1451-1469.

78 Weissman Z, Kornitzer D. A family of Candida cell surface haembinding proteins involved in haemin and haemoglobin-iron utilization. Mol Microbiol 2004; 53: 1209-1220.

79 Kaplan J, McVey Ward D, Crisp RJ, Philpott CC. Iron-dependent metabolic remodeling in S. cerevisiae. Biochim Biophys Acta 2006; 1763: 646-651.
80 Sosinska GJ, de Groot PW, Teixeira de Mattos MJ, et al. Hypoxic conditions and iron restriction affect the cell-wall proteome of Candida albicans grown under vagina-simulative conditions. Microbiology 2008; 154: (Pt 2): 510-520.

81 MacPherson S, Akache B, Weber S, et al. Candida albicans zinc cluster protein Upc2p confers resistance to antifungal drugs and is an activator of ergosterol biosynthetic genes. Antimicrob Agents Chemother. 2005; 49: 1745-1752.

82 Silver PM, Oliver BG, White TC. Role of Candida albicans transcription factor Upc2p in drug resistance and sterol metabolism. Eukaryot Cell 2004; 3: 1391-1397.

83 Hoot SJ, Oliver BG, White TC. Candida albicans UPC2 is transcriptionally induced in response to antifungal drugs and anaerobicity through Upc2p-dependent and -independent mechanisms. Microbiology 2008; 154: (Pt 9): 2748-2756.

84 Znaidi S, Weber S, Al-Abdin OZ, et al. Genomewide location analysis of Candida albicans Upc2p, a regulator of sterol metabolism and azole drug resistance. Eukaryot Cell 2008; 7: 836-847.

85 Bourot $\mathrm{S}$, Karst F. Isolation and characterization of the Saccharomyces cerevisiae SUT1 gene involved in sterol uptake. Gene 1995; 165: 97-102.

86 Malloy PJ, Zhao X, Madani ND, Feldman D. Cloning and expression of the gene from Candida albicans that encodes a high-affinity corticosteroid-binding protein. Proc Natl Acad Sci USA 1993; 90: 1902-1906.

87 Hughes AL, Todd BL, Espenshade PJ. SREBP pathway responds to sterols and functions as an oxygen sensor in fission yeast. Cell 2005; 120: 831-842.

88 Briggs MR, Yokoyama C, Wang X, Brown MS, Goldstein JL. Nuclear protein that binds sterol regulatory element of low density lipoprotein receptor promoter. I. Identification of the protein and delineation of its target nucleotide sequence. J Biol Chem 1993; 268: 14490-14496.

89 Wang X, Briggs MR, Hua X, et al. Nuclear protein that binds sterol regulatory element of low density lipoprotein receptor promoter. II. Purification and characterization. J Biol Chem 1993; 268: 14497-14504.

90 Rawson RB. The SREBP pathway - insights from Insigs and insects. Nat Rev Mol Cell Biol 2003; 4: 631-640.

91 Espenshade PJ. SREBPs: sterol-regulated transcription factors. J Cell Sci 2006; 119: (Pt 6):973-976.

92 Espenshade PJ, Hughes AL. Regulation of sterol synthesis in eukaryotes. Annu Rev Genet 2007; 41: 401-427.

93 Yang T, Espenshade PJ, Wright ME, et al. Crucial step in cholesterol homeostasis: sterols promote binding of SCAP to INSIG-1, a membrane protein that facilitates retention of SREBPs in ER. Cell 2002; 110: 489-500.

94 Brown AJ, Sun L, Feramisco JD, Brown MS, Goldstein JL. Cholesterol addition to ER membranes alters conformation of SCAP, the SREBP escort protein that regulates cholesterol metabolism. Mol Cell 2002; 10: 237-245.

95 DeBose-Boyd RA, Brown MS, Li WP, et al. Transport-dependent proteolysis of SREBP: relocation of site-1 protease from Golgi to ER obviates the need for SREBP transport to Golgi. Cell 1999; 99: 703-712.

96 Todd BL, Stewart EV, Burg JS, Hughes AL, Espenshade PJ. Sterol regulatory element binding protein is a principal regulator of anaerobic gene expression in fission yeast. Mol Cell Biol 2006; 26: 2817-2831.

97 Hughes BT, Espenshade PJ. Oxygen-regulated degradation of fission yeast SREBP by Ofd1, a prolyl hydroxylase family member. Embo $J$ 2008; 27: 1491-1501. 
98 Chen S, Sorrell T, Nimmo G, et al. Epidemiology and host- and variety-dependent characteristics of infection due to Cryptococcus neoformans in Australia and New Zealand. Australasian Cryptococcal Study Group. Clin Infect Dis 2000; 31: 499-508.

99 Kwon-Chung KJ, Bennett J.E. Medical Mycology. Philadelphia, PA: Lea \& Febiger, 1992.

100 Chun CD, Liu OW, Madhani HD. A link between virulence and homeostatic responses to hypoxia during infection by the human fungal pathogen Cryptococcus neoformans. PLoS Pathog 2007; 3: e22.

101 Himmelreich U, Dzendrowskyj TE, Allen C, et al. Cryptococcomas distinguished from gliomas with MR spectroscopy: an experimental rat and cell culture study. Radiology 2001; 220: 122-128.

102 Chang YC, Bien CM, Lee H, Espenshade PJ, Kwon-Chung KJ. Sre1p, a regulator of oxygen sensing and sterol homeostasis, is required for virulence in Cryptococcus neoformans. Mol Microbiol 2007; 64: 614-629.

103 Lee H, Bien CM, Hughes AL, et al. Cobalt chloride, a hypoxiamimicking agent, targets sterol synthesis in the pathogenic fungus Cryptococcus neoformans. Mol Microbiol 2007; 65: 1018-1033.

104 Goldberg MA, Glass GA, Cunningham JM, Bunn HF. The regulated expression of erythropoietin by two human hepatoma cell lines. Proc Natl Acad Sci USA 1987; 84: 7972-7976.

105 Wang GL, Semenza GL. General involvement of hypoxia-inducible factor 1 in transcriptional response to hypoxia. Proc Natl Acad Sci USA 1993; 90: 4304-4308.

106 Wang G, Hazra TK, Mitra S, Lee HM, Englander EW. Mitochondrial DNA damage and a hypoxic response are induced by $\mathrm{CoCl}[2)$ in rat neuronal PC12 cells. Nucleic Acids Res 2000; 28: 2135-2140.

107 Huang Y, Du KM, Xue ZH, et al. Cobalt chloride and low oxygen tension trigger differentiation of acute myeloid leukemic cells: possible mediation of hypoxia-inducible factor-1alpha. Leukemia 2003; 17: 2065-2073.

108 Grasselli F, Basini G, Bussolati S, Bianco F. Cobalt chloride, a hypoxiamimicking agent, modulates redox status and functional parameters of cultured swine granulosa cells. Reprod Fertil Dev 2005; 17: $715-72$.

109 Ingavale SS, Chang YC, Lee H, et al. Importance of mitochondria in survival of Cryptococcus neoformans under low oxygen conditions and tolerance to cobalt chloride. PLoS Pathog 2008; 4: e1000155.

110 Bahn YS, Kojima K, Cox GM, Heitman J. A unique fungal twocomponent system regulates stress responses, drug sensitivity, sexual development, and virulence of Cryptococcus neoformans. Mol Biol Cell 2006; 17: 3122-3135.

This paper was first published online on Early Online on 22 May 2009.
111 Millner PD, Marsh PB, Snowden RB, Parr JF. Occurrence of Aspergillus fumigatus during composting of sewage sludge. Appl Environ Microbiol 1977; 34: 765-772.

112 Tekaia F, Latge JP. Aspergillus fumigatus: saprophyte or pathogen? Curr Opin Microbiol 2005; 8: 385-392.

113 Wilson DM, Mubatanhema W, Jurjevic Z. Biology and ecology of mycotoxigenic Aspergillus species as related to economic and health concerns. Adv Exp Med Biol 2002; 504: 3-17.

114 Latge JP. Aspergillus fumigatus and aspergillosis. Clin Microbiol Rev 1999; 12: 310-350.

115 Perfect JR, Cox GM, Lee JY, et al. The impact of culture isolation of Aspergillus species: a hospital-based survey of aspergillosis. Clin Infect Dis 2001; 33: 1824-1833.

116 Latge JP. The pathobiology of Aspergillus fumigatus. Trends Microbiol 2001; 9: 382-389.

117 Rhodes JC. Aspergillus fumigatus: growth and virulence. Med Mycol 2006; 44: (Suppl. 1): S77-81.

118 Hohl TM, Feldmesser M. Aspergillus fumigatus: principles of pathogenesis and host defense. Eukaryot Cell. 2007; 6:1953-1963.

119 Tarrand JJ, Lichterfeld M, Warraich I, et al. Diagnosis of invasive septate mold infections. A correlation of microbiological culture and histologic or cytologic examination. Am J Clin Pathol 2003; 119: 854-858.

120 Tarrand JJ, Han XY, Kontoyiannis DP, May GS. Aspergillus hyphae in infected tissue: evidence of physiologic adaptation and effect on culture recovery. J Clin Microbiol 2005; 43: 382-386.

121 Taubitz A, Bauer B, Heesemann J, Ebel F. Role of respiration in the germination process of the pathogenic mold Aspergillus fumigatus. Curr Microbiol 2007; 54: 354-360.

122 Lockington RA, Borlace GN, Kelly JM. Pyruvate decarboxylase and anaerobic survival in Aspergillus nidulans. Gene 1997; 191: 61-67.

123 Kelly JM, Drysdale MR, Sealy-Lewis HM, Jones IG, Lockington RA. Alcohol dehydrogenase III in Aspergillus nidulans is anaerobically induced and post-transcriptionally regulated. Mol Gen Genet 1990; 222: 323-328.

124 Willger SD, Puttikamonkul S, Kim KH, et al. A sterol-regulatory element binding protein is required for cell polarity, hypoxia adaptation, azole drug resistance, and virulence in Aspergillus fumigatus. PLoS Pathog 2008; 4: e1000200.

125 Hughes AL, Lee CY, Bien CM, Espenshade PJ. 4-Methyl sterols regulate fission yeast SREBP-Scap under low oxygen and cell stress. J Biol Chem 2007; 282: 24388-24396. 